\title{
Analysis of crucial genes, pathways and construction of the molecular regulatory networks in vascular smooth muscle cell calcification
}

\author{
XIAOMIN WEI, YIMING SU, QIYI LI, ZHIYONG ZHENG and PEIYONG HOU \\ Department of Vascular Surgery, Liuzhou Worker's Hospital, The Fourth Affiliated Hospital \\ of Guangxi Medical University, Liuzhou, Guangxi 545005, P.R. China \\ Received September 12, 2020; Accepted February 11, 2021
}

DOI: $10.3892 /$ etm.2021.10021

\begin{abstract}
Vascular calcification (VC) accompanies the trans-differentiation of vascular smooth muscle cells (VSMCs) into osteo/chondrocyte-like cells and resembles physiological bone mineralization. However, the molecular mechanisms underlying VC initiation and progression have remained largely elusive. The aim of the present study was to identify the genes and pathways common to VSMC and osteoblast calcification and construct a regulatory network of non-coding RNAs and transcription factors (TFs). To this end, the Gene Expression Omnibus dataset GSE37558 including mRNA microarray data of calcifying VSMCs (CVSMCs) and calcifying osteoblasts (COs) was analyzed. The differentially expressed genes (DEGs) were screened and functionally annotated and the microRNA (miRNA/mRNA)-mRNA, TF-miRNA and long non-coding RNA (lncRNA)-TF regulatory networks were constructed. A total of 318 DEGs were identified in the CVSMCs relative to the non-calcified VSMCs, of which 43 were shared with the COs. The CVSMC-related DEGs were mainly enriched in the functional terms cell cycle, extracellular matrix (ECM), inflammation and chemotaxis-mediated signaling pathways, of which ECM was enriched by the DEGs for the COs as well. The protein-protein interaction network of CVSMCs consisted of 281 genes and 3,650 edges. There were 30 hub genes in this network, including maternal embryonic leucine zipper kinase (MELK), which potentially regulates the differentially expressed TF (DETF) forkhead box (FOX)M1
\end{abstract}

Correspondence to: Professor Peiyong Hou, Department of Vascular Surgery, Liuzhou Worker's Hospital, The Fourth Affiliated Hospital of Guangxi Medical University, 156 Heping Road, Liuzhou, Guangxi 545005, P.R. China

E-mail: hPeiYong@126.com

Abbreviations: VC, vascular calcification; CVSMCs, calcifying vascular smooth muscle cells; CO, calcifying osteoblast; DEGs, differentially expressed genes; GO, Gene Ontology

Key words: VSMC calcification, osteoblast, lncRNA, miRNA, transcription factor, bioinformatics and is a potential target gene of Homo sapiens miR-485-3p and miR-181d. The TF-miRNA network included 251 TFs and 60 miRNAs, including 10 DETFs such as FOXO1 and snail family transcriptional repressor 2 (SNAI2). Furthermore, the lncRNAs H19 imprinted maternally expressed transcript (H19) and differentiation antagonizing non-protein coding RNA (DANCR) were predicted as the upstream regulators of FOXO1 and SNAI2 in the lncRNA-TF regulatory network. DANCR, MELK and FOXM1 were downregulated, and H19, FOXO1 and SNAI2 were upregulated in the CVSMCs. Taken together, the CVSMCs and COs exhibited similar molecular changes in the ECM. In addition, the MELK-FOXM1, H19/DANCR-FOXO1 and SNAI2 regulatory pathways likely mediate VSMC calcification.

\section{Introduction}

Vascular calcification (VC) refers to the ectopic deposition of calcium phosphate crystals or hydroxyapatite on the vascular walls. It is frequently observed during ageing, as well as in degenerative diseases such as chronic kidney disease (CKD), diabetes and atherosclerosis, and significantly increases the risk of cardiovascular disease (CVD) and mortality (1-3). Contrary to the long-held surmise that calcium and phosphorus are passively deposited on the vascular walls, recent studies have indicated that $\mathrm{VC}$ is an active and cell-regulated process similar to the mineralization of osteo/chondrocyte-like cells during bone formation (4). The incidence of coronary artery calcification in CKD patients is $>50 \%$ in the absence of dialysis and significantly higher at $70-90 \%$ among patients undergoing dialysis (5). In addition, increased calcium burden of the thoracic aorta elevates the risk of CVD by 3.7-fold (6), whereas abdominal aorta calcification increases the relative risk of coronary, cerebrovascular and cardiovascular events as well as mortality rates (7). VC is a significant risk factor of CVD in $90 \%$ of males and $67 \%$ of females above the age of 70 years (8).

Vascular smooth muscle cells (VSMCs) may differentiate into the major cell types in the vessel wall in response to suitable environmental stimuli. There is evidence that VSMCs lose their contractile phenotype and trans-differentiate into osteoblast-like cells expressing osteogenic transcription factors and proteins. These cells may initiate calcification of the vascular 
wall by secreting calcium and phosphorous-loaded exosomes into the extracellular matrix (ECM) (9-11). However, little is known regarding the specific pathways and molecular mechanisms underlying VC, which markedly limits the development of effective drugs.

Non-coding RNAs are a class of transcripts that regulate the expression of protein-encoding genes through various mechanisms. MicroRNAs (miRNAs/miRs) are 18-22 nucleotides in length and regulate target gene expression at the post-transcriptional level through binding at the 3 ' untranslated region. Long non-coding RNAs (lncRNAs) are >200 nucleotides long and exert their regulatory effects through more complex mechanisms (12). Several lncRNAs have been identified in recent years that are involved in the progression of various pathological conditions, including CVD (13-15). Furthermore, specific miRNAs and lncRNAs have been implicated in VSMC calcification. For instance, downregulation of miR-204, miR-29b or miR-30e trigger the osteogenic differentiation and calcification of VSMCs both in vitro and in vivo, whereas upregulation of miRNA-128 accelerates cardiovascular calcification (16-20). Lin et al (21) demonstrated that IncRNA-ES3 enhanced hyperglycemia-induced calcification of VSMCs by suppressing miR-34c-5p expression as a sponge. In addition, Jeong et al (22) identified numerous differentially expressed lncRNAs in calcified rat VSMCs, of which leucine rich repeat containing $75 \mathrm{a}$-antisense (AS)1 was significantly downregulated and its ectopic expression attenuated calcium accumulation in VSMCs cultured with inorganic phosphate. However, the exact mechanistic roles of non-coding RNAs in VSMC calcification have remained to be elucidated.

In the present study, the differentially expressed genes (DEGs) and pathways in VSMCs exposed to high and normal calcium levels for varying durations were identified using bioinformatics. Given the physiological similarities between VCs and bone mineralization, the DEGs and pathways common to both calcifying VSMCs (CVSMCs) and osteoblasts (COs) were also screened and certain potentially crucial genes were experimentally validated. The putative regulatory networks of non-coding RNAs and transcription factors (TFs) in CVSMCs were also predicted. The present results provide novel insight into the molecular basis of the pathogenesis of $\mathrm{VC}$.

\section{Materials and methods}

Microarray data and identification of DEGs. The microarray dataset GSE37558 profiled on the GPL6947 platform (Illumina HumanHT-12 V3.0 expression beadchip) was downloaded from the National Center for Biotechnology Information (NCBI)-Gene Expression Omnibus database (https://www.ncbi.nlm.nih.gov/geo/query/acc.cgi). It includes the mRNA expression data of $32 \mathrm{VSMCs}$ and osteoblasts samples cultured for $0,2,8,12$ or 25 days (3-4 replicates per time-point) in calcified medium containing $1.8 \mathrm{mM} \mathrm{Ca}^{2+}(23)$. The raw data were integrated and the DEGs between the control and calcified VSMCs were identified using the GEO2R tool, which is a GEO tool (https://www.ncbi.nlm. nih.gov/geo/geo2r/?acc=GSE37558) and Morpheus website (https://software.broadinstitute.org) using an adjusted $\mathrm{P}<0.05$ with $\mid \log$ fold changel $>1$ as the thresholds. The DEGs common to VSMCs calcified for varying durations were also identified
Table I. Primer sequences or numbers for PCR.

Gene

symbol Primer sequence (5'-3') or catalogue number

DANCR Forward: CAGCTGACCCTTACCCTGAA

Reverse: GACCCTGGGGTTGTTAGTCA

H19 Forward: CAGAGTCCGTGGCCAAGG

Reverse: CGCCTTCAGTGACTGGCA

MELK Forward: ACTGCCCTGGAGGAGAGCT

Reverse: AGCCCTGGCTGTGCACATAA

FOXM1 Forward: GGAGGAAATGCCACACTTAGCG

Reverse: TAGGACTTCTTGGGTCTTGGGGTG

FOXO1 Forward: TACGCCGACCTCATCACCAAG'

Reverse: GCACGCTCTTCACCATCCACT'

SNAI2 Forward: CACCTCCTCCAAGGACCA'

Reverse: GGCCAGCCCAGAAAAAGT

GAPDH Forward: GTCAGCCGCATCTTCTTT'

Reverse: CGCCCAATACGACCAAAT'

U6

Hsa-miR-

RQP086799

485-3p

Hsa- miR-

HmiRQP0521

181d-5p

HmiRQP0237

Hsa, Homo sapiens; miR, microRNA; FOX, forkhead box; DANCR, differentiation antagonizing non-protein coding RNA; H19, H19 imprinted maternally expressed transcript; MELK, maternal embryonic leucine zipper kinase; SNAI2, snail family transcriptional repressor 2 .

using the same criteria. The DEGs in osteoblasts were similarly screened, and the shared DEGs between CVSMCs and COs were also defined using the Morpheus website.

Gene ontology $(G O)$ and pathway enrichment analysis. The DEGs were functionally annotated by GO, Kyoto Encyclopedia of Genes and Genomes (KEGG) pathway and reactome analyses using the Database for Annotation, Visualization and Integrated Discovery version 6.8 (https://david.ncifcrf.gov/). The significantly enriched genes or pathways were screened on the basis of $\mathrm{P}<0.05$.

Protein-protein interaction (PPI) network. The PPI networks of the DEGs were constructed using a Search Rool for the Retrieval of Interacting Genes and proteins (STRING) database (http://string.embl.de/; accessed April, 2019) (24) in order to identify the interacting and hub genes. Cytoscape software (25) was used for visualizing the networks and analyzing the degree of connectivity of nodes. Finally, module analysis of the PPI network was performed using the molecular complex detection application of Cytoscape software. The module genes were functionally annotated as described above.

Construction of regulatory networks. The target miRNAs of calcification-related DEGs were predicted using the miRWalk (http://zmf.umm.uni-heidelberg.de; 

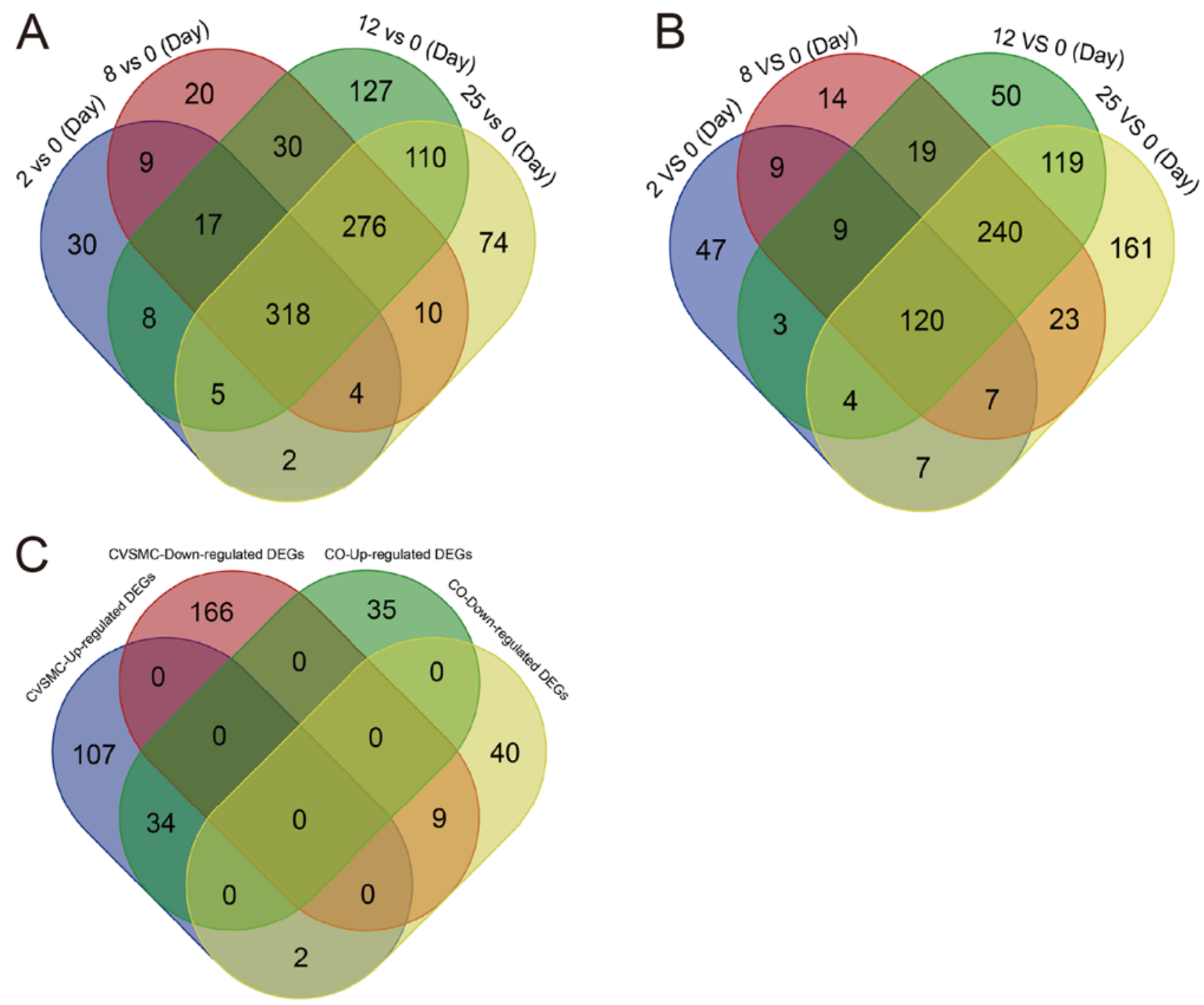

Figure 1. Identification of DEGs in CVSMCs and COs. (A) VSMCs cultured in high-calcium medium for 2, 8, 12 and 25 days had 318 DEGs relative to the 0 -day normal control. The DEGs observed at the different time-points of calcification are color-coded. The overlapping regions represent shared DEGs across the different time-points. (B) A total of 43 DEGs were common between CVSMCs and COs, including 34 upregulated and 9 downregulated genes, which were color-coded. (C) Osteoblasts cultured for 2, 8, 12 and 25 days in calcification medium had 206, 441, 565 and 681 DEGs compared to the normal osteoblasts, respectively, and 120 DEGs were consistent across all time-points. The DEGs from different groups are color-coded and the overlapping region represents shared DEGs. VC, vascular calcification; CVSMCs, calcifying vascular smooth muscle cells; CO, calcifying osteoblast; DEGs, differentially expressed genes; GO, Gene Ontology.

accessed April, 2019), miRanda (http://www.microrna. org/microrna/home.do; accessed April, 2019), miRDB (http://www.mirdb.org/; accessed April, 2019), RNA22 (https://cm.jefferson.-edu/rna22/; accessed April, 2019) and TargetScan (http://www.targetscan.org/vert_72/; accessed April, 2019) databases. The miRNA-target DEG pairs were established when predicted by all five databases. The TFs targeting miRNAs in the miRNA-target gene network were predicted using the TransmiR (http://www.cuilab.cn/transmir; accessed April, 2019) database (26) based on literature-curated TF-miRNA regulation data. The predicted TFs within the DEGs were defined as differentially expressed TFs (DETFs) and used to construct the DETF-miRNA-target DEG network. In addition, the TF-miRNA-hub gene network was also established using hub genes identified from the PPI network. Finally, the lncRNAs targeting DETFs in the TF-miRNA-hub gene network were screened from the LncRNA2Target v2.0 database (http://123.59.132.21/lncrna2target; accessed April,
2019) (27) to construct the IncRNA-DETF-miRNA-target gene network. All regulatory networks were established and visualized using Cytoscape.

Cell culture and calcification assay. Human VSMC line (cat. no. CRL-1999) was purchased from Aolu Biotech and cultured in Dulbecco's modified Eagle's medium (Gibco; Thermo Fisher Scientific, Inc.) containing $10 \%$ fetal bovine serum (FBS; Gibco; Thermo Fisher Scientific, Inc.) and $1 \%$ penicillin-streptomycin (Beyotime Institute of Biotechnology) at $37^{\circ} \mathrm{C}$ under $5 \% \mathrm{CO}_{2}$. The cells were harvested at $80-90 \%$ confluency using $0.25 \%$ trypsin/0.02\% EDTA solution (Beyotime Institute of Biotechnology) and cells from passages 3-7 were used in the experiments. For in vitro calcification, VSMCs were seeded in 6-well plates with 60-70\% cell density and cultured in growth medium containing $1.8 \mathrm{mM} \mathrm{CaCl}_{2}$ (Sigma-Aldrich; Merck KGaA) for 12 days. The medium was replaced every 2-3 days. 


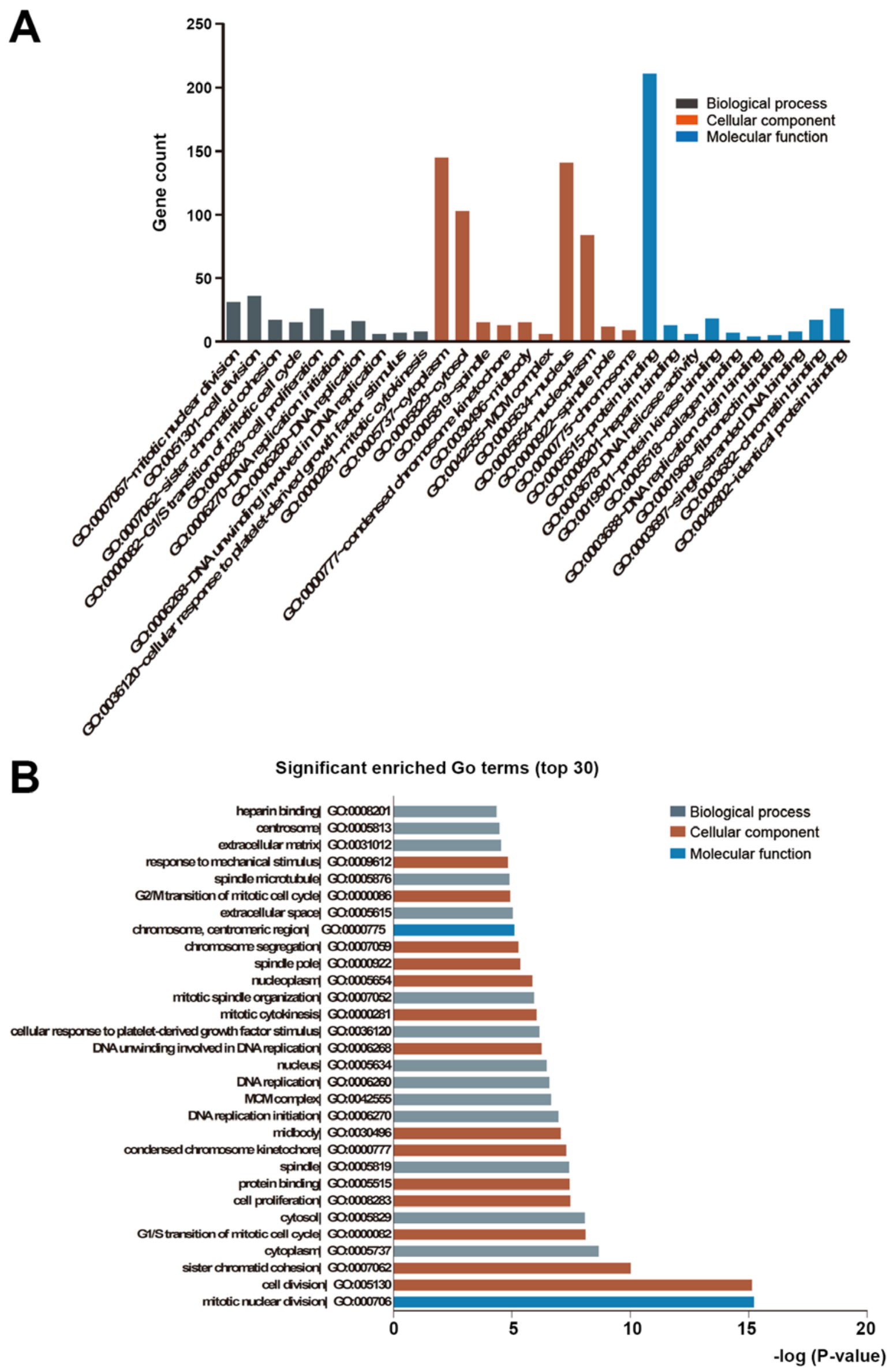

Figure 2. GO term enrichment analysis of DEGs from CVSMCs. (A) GO terms related to molecular function, biological process and cellular component enriched in the DEGs from CVSMCs. (B) Top $30 \mathrm{GO}$ terms for DEGs from CVSMCs. CVSMCs, calcifying vascular smooth muscle cells; DEGs, differentially expressed genes; GO, Gene Ontology. 


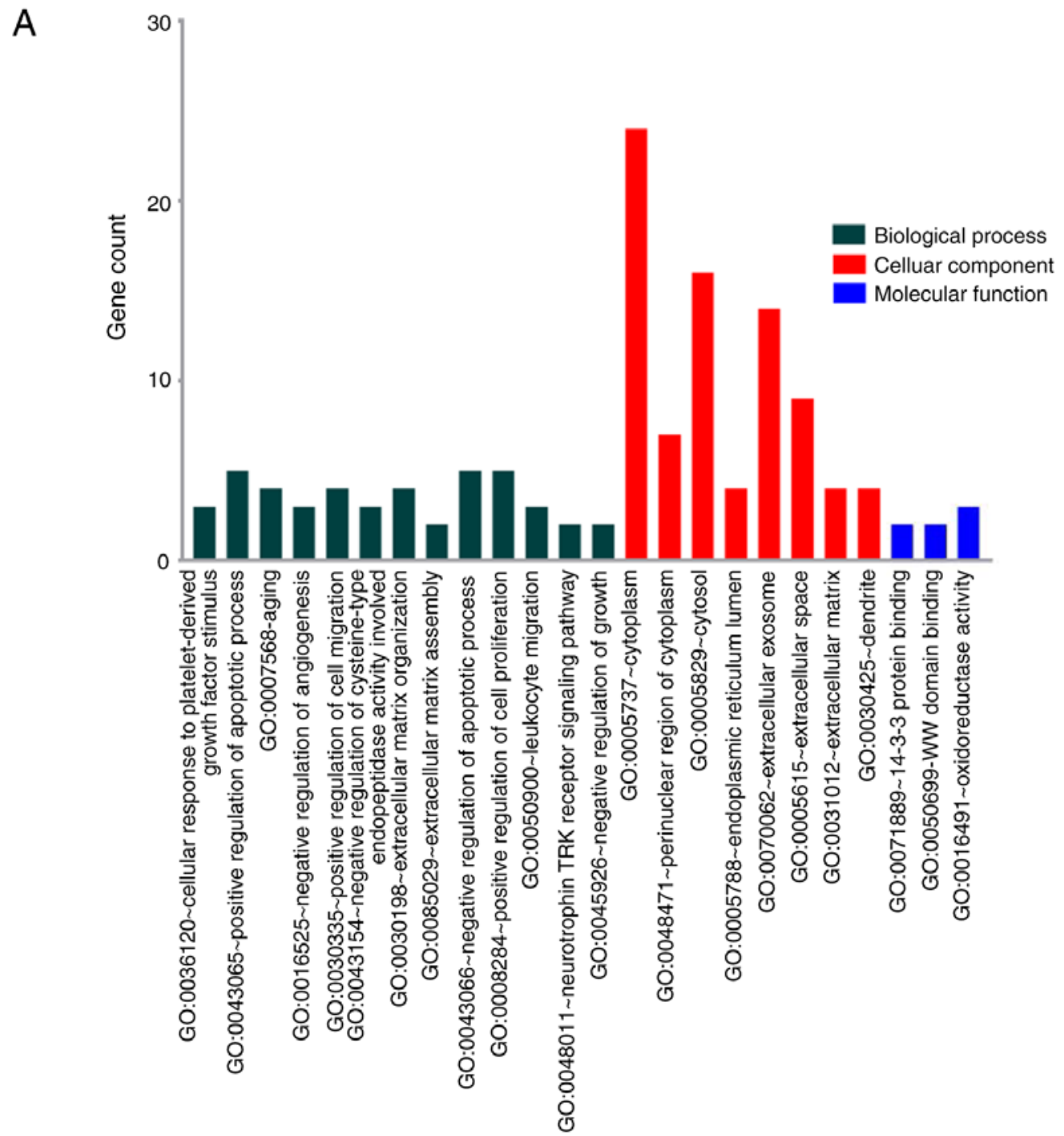

B

Significant enriched GO terms (Top 30)

GO:0071158 positive regulation of cell cycle arrest GO:0071889 14-3-3 protein binding GO:0030425 dendrite GO:0071294 cellular response to zinc ion GO:0044849 estrous cycle

GO:0048011 neurotrophin TRK receptor signaling pathway GO:0045892 negative regulation of transcription, DNA-templated GO:0050900 leukocyte migration GO:0031012 extracellular matrix GO:0008284 positive regulation of cell proliferation GO:0010628 positive regulation of gene expression GO:0085029 extracellular matrix assembly GO:0005615 extracellular space GO:0070062 extracellular exosome GO:0030198 extracellular matrix organization GO:0043154 negative regulation of cysteine-type endopeptidase activity involved
GO:0042133 neurotransmitter metabolic process GO:0042133 neurotransmitter metabolic process
GO:0030335 positive regulation of cell migration GO:0005788 endoplasmic reticulum lumen

GO:0016525 negative regulation of angiogenesis GO:0005829 cytosol GO:0007568 aging GO:1900625 positive regulation of monocyte aggregation GO:0043065 positive regulation of apoptotic process GO:0048471 perinuclear region of cytoplasm GO:0005737 cytoplasm GO:0007155 cell adhesion
-

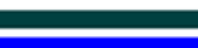

-

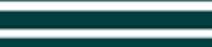
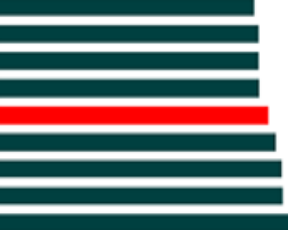

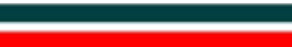

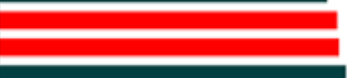

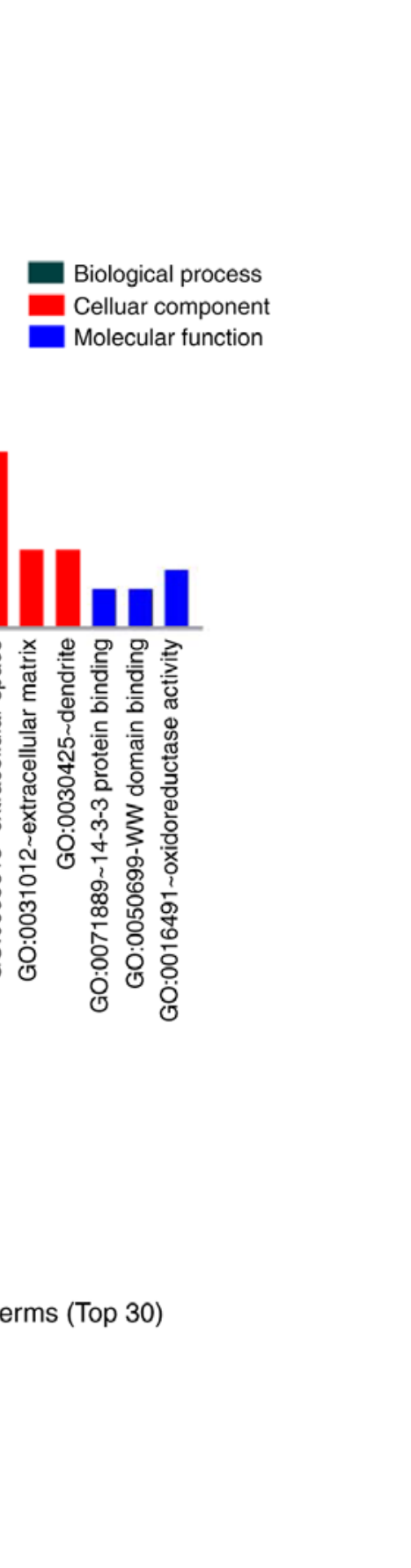

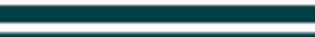

(2)
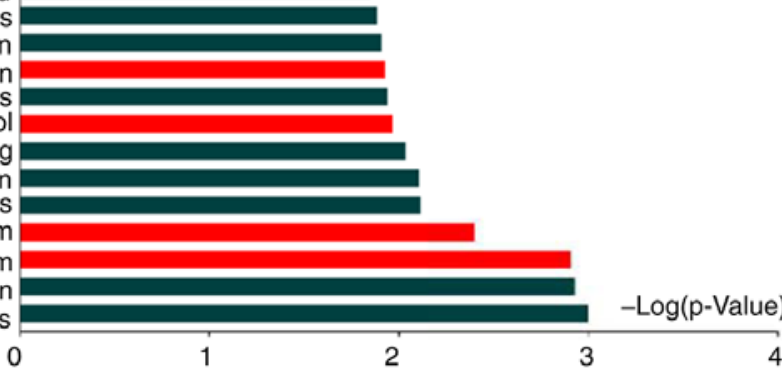

GO:0036120 cellular response to platelet-derived growth factor stimulus

1

Biological process

Celluar component

Molecular function

Figure 3. GO analysis of DEGs shared between CVSMCs and CO. (A) GO terms of DEGs in CVSMCs and CO according to molecular function, biological process and cellular component. (B) Top $30 \mathrm{GO}$ terms for DEGs common to CVSMCs and COs. CVSMCs, calcifying vascular smooth muscle cells; CO, calcifying osteoblast; DEGs, differentially expressed genes; GO, Gene Ontology. 
Significant enriched pathway terms (Top 30)

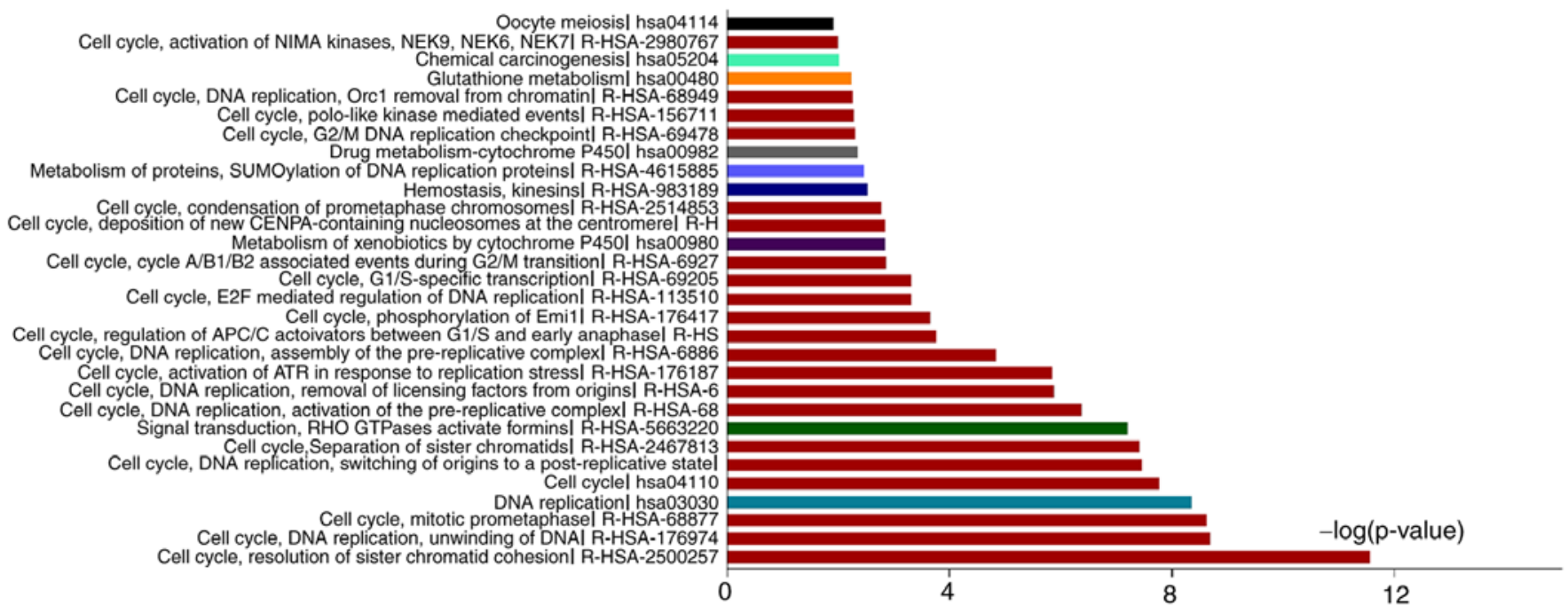

Figure 4. Top 30 signaling pathways for calcification-related differentially expressed genes in calcifying vascular smooth muscle cells determined using Kyoto Encyclopedia of Genes and Genomes pathway analysis and Reactomen online dataset. Hsa, Homo sapiens.

$R N A$ extraction and reverse transcription-quantitative $(R T-q)$ $P C R$ analysis. TRIzol ${ }^{\circledR}$ (Invitrogen; Thermo Fisher Scientific, Inc.) was used to extract total RNA from the cultured VSMCs according to the manufacturer's protocol. The integrity of the isolated RNA was determined by measuring the absorbance at $260 \mathrm{~nm}$ (A260) and the purity in terms of the A260/A280 ratio. Total RNA was reverse-transcribed into complementary (c)DNA using the TransScript kit (Takara Biotechnology, Inc.). qPCR was performed with $2 \mu \mathrm{l}$ first-strand cDNA as the template and the FastStart SYBR Green kit (Roche). The primers were purchased from GeneCopoeia Biotechnology Company and their sequences are presented in Table I. The thermocycling conditions of qPCR were as follows: $2 \mathrm{~min}$ at $50^{\circ} \mathrm{C}$ and $10 \mathrm{~min}$ at $95^{\circ} \mathrm{C}$, followed by 40 cycles of $15 \mathrm{sec}$ at $95^{\circ} \mathrm{C}, 30 \mathrm{sec} 60^{\circ} \mathrm{C}$ and $30 \mathrm{sec}$ at $72^{\circ} \mathrm{C}$ (ABI 7500 Fast; Applied Biosystems; Thermo Fisher Scientific, Inc.). GAPDH and U6 were used as the internal controls for mRNAs and miRNAs, respectively. The relative expression of these genes was determined using the comparative CT $\left(2^{-\Delta \Delta \mathrm{Cq}}\right)$ method (28).

Statistical analysis. The in vitro experiments were performed in triplicate and were independently repeated three times. SPSS version 21.0 (IBM Corp.) was used for statistical analysis. Data were expressed as the mean \pm standard deviation and compared using an unpaired t-test. $\mathrm{P}<0.05$ was considered to indicate statistical significance.

\section{Results}

Identification of calcification-related DEGs in the CVSMCs and COs. To identify the genes affected by short- and long-term high $\mathrm{Ca}^{2+}$ exposure, VSMCs were cultured in the calcification medium for varying durations and DEGs were detected (GSE37558). As presented in Fig. 1A, VSMCs cultured for 2, 8, 12 and 24 h had 393, 684, 892 and 799 DEGs relative to the normal cells ( 0 -day control), respectively. Furthermore, 318 genes were consistently differentially expressed across all time-points, including 147 upregulated and 185 downregulated genes (Fig. 1A, Tables SI and SII). Furthermore, there were 206, 441, 565 and 681 DEGs in the osteoblasts cultured for 2, 8, 12 and 25 days, respectively, in the calcification medium compared to normal osteoblasts, of which 120 DEGs were common to all time-points (Fig. 1B, Tables SIII and SIV). Furthermore, 43 DEGs were common to the CVSMCs and COs, including 34 upregulated and 9 downregulated genes (Fig. 1C).

Functional annotation of calcification-related DEGs. The common DEGs (see above) were functionally annotated in GO terms and KEGG pathways. The upregulated genes were significantly enriched in biological process terms of ECM organization, cell adhesion, positive regulation of apoptotic process and positive regulation of gene expression, whereas the downregulated DEGs were significantly enriched in cell division, mitotic nuclear division, cell proliferation and cell cycle. The significant molecular function terms for upregulated genes were integrin, heparin, receptor and fibronectin binding, while those for downregulated genes were protein, ATP, protein kinase and chromatin binding. Finally, in the category cellular component, the upregulated genes were mainly enriched in terms such as cytoplasm, extracellular space, extracellular exosome, ECM and extracellular region, while the downregulated genes were mainly related to the nucleoplasm, nucleus, cytosol and cytoplasm (Fig. 2A and B, Table SV). GO analysis of the shared DEGs between CVSMCs and CO revealed cytoplasm, ECM/exosome/space and tyrosine metabolism as the enriched terms (Fig. 3A and B, Table SVI). These results indicate that the functional clusters of these DEGs are closely related to the cell cycle, ECM and cell binding. Furthermore, the ECM has an important role in both VSMC and osteoblast calcification.

The significantly enriched pathways of upregulated genes were platelet-derived growth factor, mineral absorption, vascular smooth muscle contraction, focal adhesion and drug 


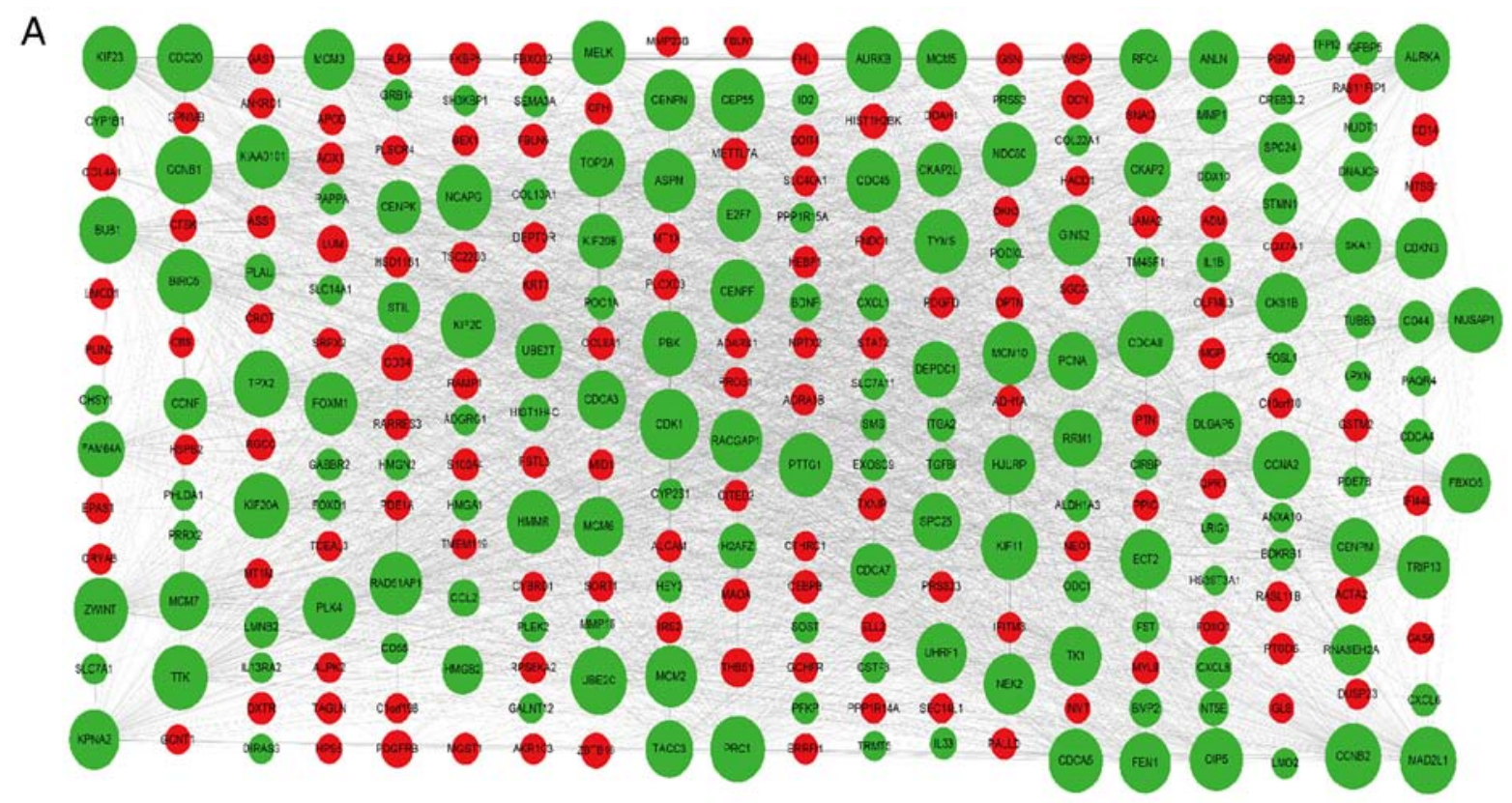

B

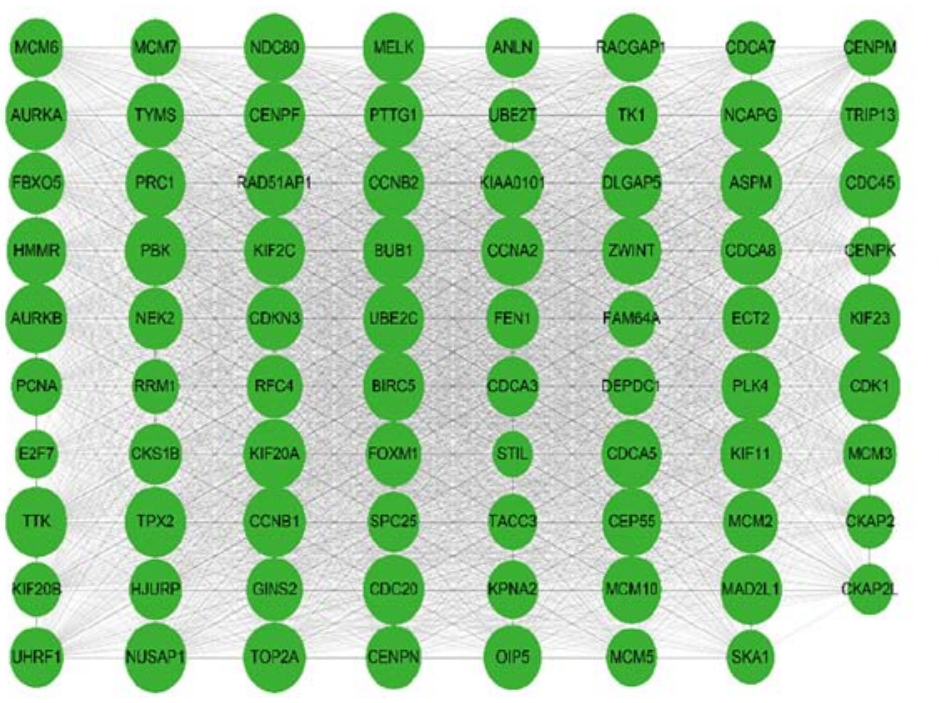

C

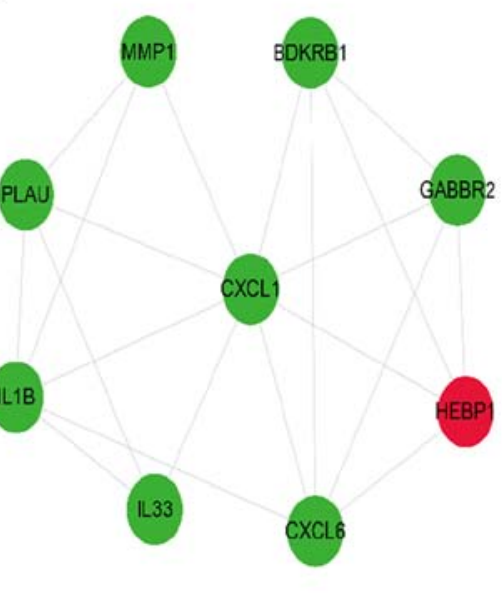

D

TGFB

$E$

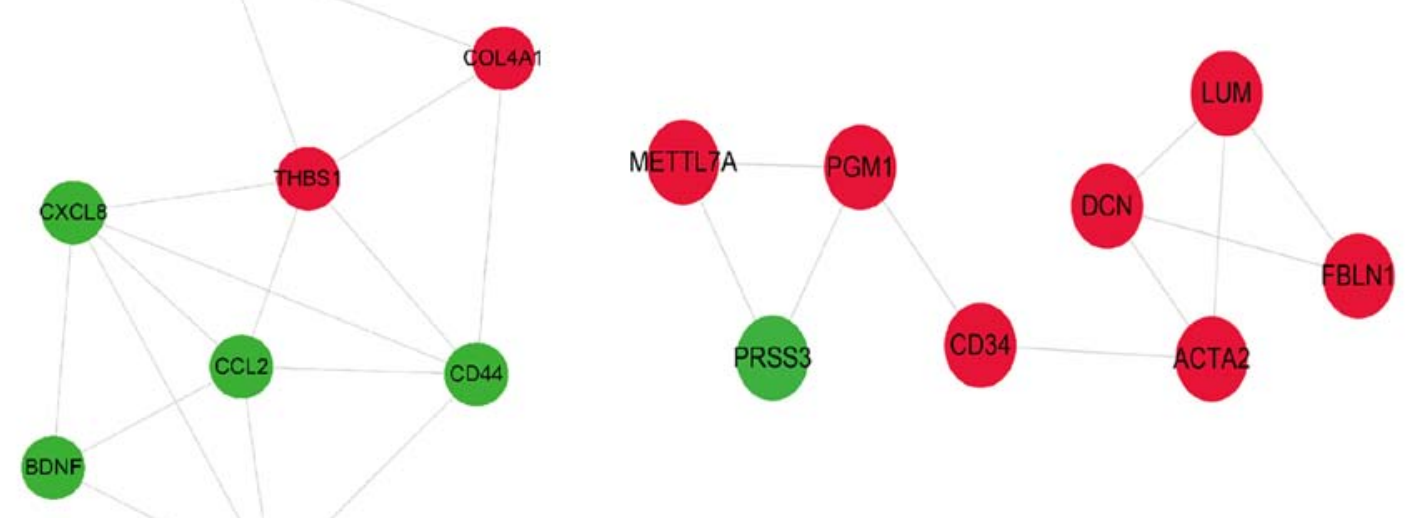

NT5E

Figure 5. PPI networks and modular analysis of DEGs. (A) PPI network of DEGs in calcifying vascular smooth muscle cells, consisting of 281 gene nodes and 3,650 edges. The red and green gene nodes represent the upregulated and downregulated genes, respectively. The volume of gene nodes is proportional to the degree of connectivity. (B) Module 1 consisted of 79 gene nodes and 2,770 edges. (C) Module 2 consisted of 9 gene nodes and 20 edges. (D) Module 3 consisted of 8 gene nodes and 16 edges. (E) Module 4 consisted of 8 gene nodes and 10 edges. Module analysis utilized the following cut-off criteria: Degree cutoff, 2; node score cutoff, 0.2 ; K-core cutoff, 2; and max depth, 100. DEGs, differentially expressed genes; PPI, protein-protein interaction. 

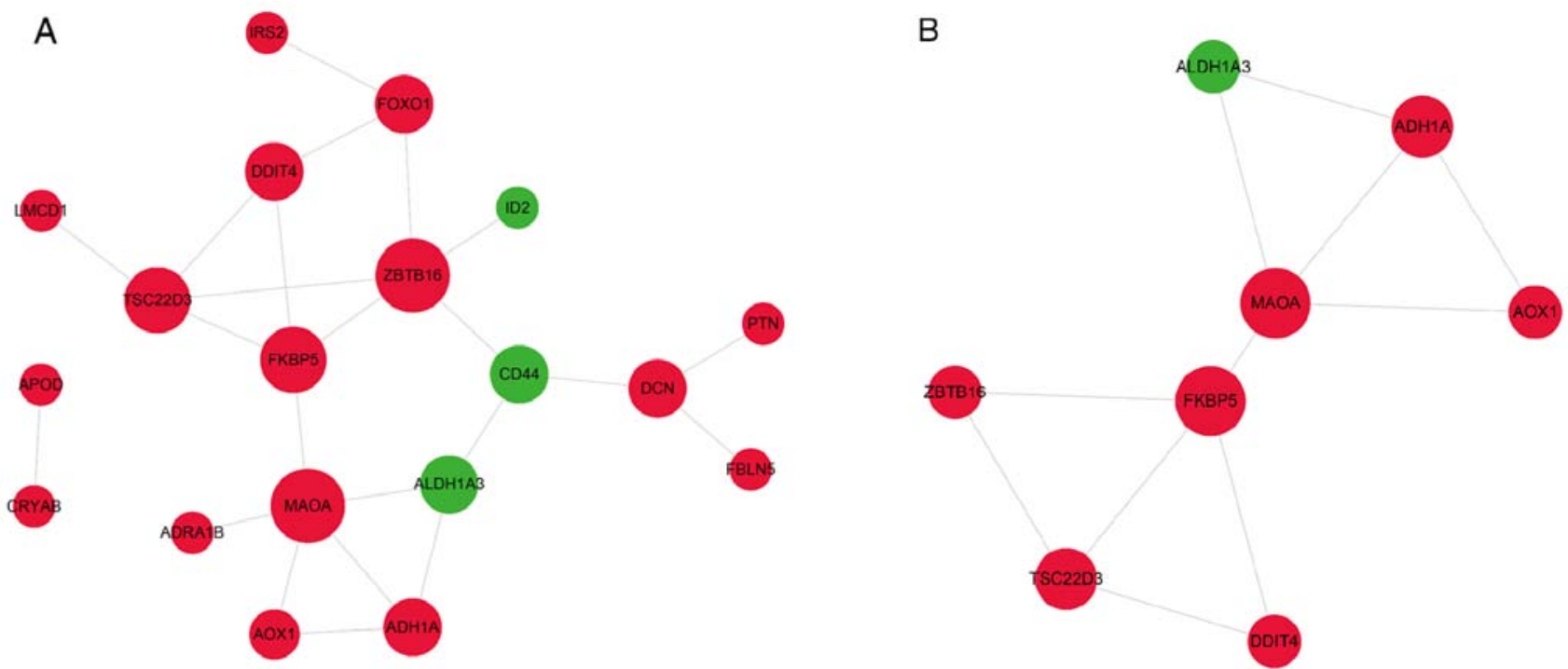

Figure 6. Protein-protein interaction of shared DEGs between calcifying vascular smooth muscle cells and calcifying osteoblasts. (A) Module 1 of the shared DEGs consisted of 19 gene nodes and 23 edges. (B) Module 2 of the shared DEGs consisted of 8 gene nodes and 11 edges. The red and green nodes represent upregulated and downregulated genes, respectively. The volume of gene nodes is proportional to the degree of connectivity. Module analysis utilized the following cut-off criteria: Degree cutoff, 2; node score cutoff, 0.2; K-core cutoff, 2; and max depth, 100. DEGs, differentially expressed genes.

metabolism-cytochrome P450, and those for downregulated DEGs were resolution of sister chromatid cohesion, mitotic prometaphase, cell cycle and separation of sister chromatids (Fig. 4, Table SVII). Finally, the DEGs common to CVMCs and COs were enriched in pathways of amino acid metabolism and metallothionein-binding metals (Table SVIII). Thus, genes related to distinct pathways were affected during calcification of VSMCs and the downregulated genes in particular were strongly associated with cell cycle and proliferation.

Identification of hub genes involved in VSMC calcification and osteoblast mineralization. The hub genes involved in the calcification of VSMCs were next identified by constructing a PPI network of the 318 common DEGs. As presented in Fig. 5A, the PPI network consisted of 281 genes, including 143 upregulated and 175 downregulated genes, and 3,650 edges. A total of 30 genes with a degree of connectivity of $>80$ in the PPI network were designated as the hub genes, including cyclin dependent kinase 1 , mitotic arrest deficient 2 like 1 (MAD2L1), kinesin family member 11 (KIF11), maternal embryonic leucine zipper kinase (MELK), non-SMC condensin I complex subunit G (NCAPG), PDZ binding kinase (PBK), kinesin family member 23 (KIF23) and cyclin A2. Module analysis of the PPI network using the MCODE app further revealed 4 modules (Fig. 5B-E). Module 1 with 79 nodes and 2,770 edges was closely associated with the cell cycle, cell division and separation of sister chromatid pathways. Module 2 consisted of 9 nodes and 20 edges and was significantly enriched in the pathways of inflammatory response, immune response, chemokine-mediated signaling and positive regulation of interleukin (IL)- 6 production (Fig. 7A and Table SIX). In addition, module 3 was comprised of 8 nodes and 16 edges and was mainly involved in ECM organization, cellular response to fibroblast growth factor stimulus, cell adhesion, chemokine-mediated signaling and NOD-like receptor signaling pathways. Finally, module 4 had 8 nodes and 10 edges, and was associated with extracellular space and ECM pathways (Fig. 7B and Table SIX). These results suggested that signaling pathways related to the cell cycle, immune response, ECM, chemotaxis and inflammatory response have a key role in VSMC calcification.

VSMCs are able to differentiate into osteoblast-like cells expressing osteogenic proteins in response to dysregulated calcium-phosphate metabolism and thus contribute to VC $(9,10)$. Therefore, the PPI network of the 43 DEGs shared by CVSMCs and COs was also established in order to identify signaling pathways involved in VSMCs and osteoblast mineralization. As presented in Fig. 6A, the PPI network contained 19 gene nodes and 23 edges. One module including 8 nodes and 11 edges (Fig. 6B) was identified, which was associated with amino acid metabolism, drug metabolism-cytochrome P450, metabolic pathways and oxidoreductase activity (Fig. 7C and Table SX).

VSMC calcification-related regulatory networks. To further explore the regulatory mechanisms involved in VSMC calcification, miRNAs targeting the CVSMC-related DEGs were predicted by the miRanda, miRDB, miRWalk, RNA22 and TargetScan databases. A total of 76 putative miRNAs and 140 miRNA-target pairs were identified. A miRNA-target regulatory network was constructed with 76 miRNAs, 53 DEGs and 140 edges, and included Homo sapiens (hsa)-miR-20a-PBK, hsa-miR-15a- kinesin family member 23 (KIF23), hsa-miR-511-snail family transcriptional repressor 2 (SNAI2), hsa-miR-507-SNAI2, hsa-miR-181d- maternal embryonic leucine zipper kinase (MELK) and hsa-miR-485-3p-MELK regulatory pairs (Fig. 8 and Table SXI). Similarly, IncRNA and TF-mediated regulatory networks were also constructed. A total of 251 TFs were predicted and the TF-miRNA regulatory network consisted of 754 miRNA-target pairs, 251 TFs and 60 target DEGs (Fig. 9A and Table SXII). The TFs FOS like 1, AP-1 


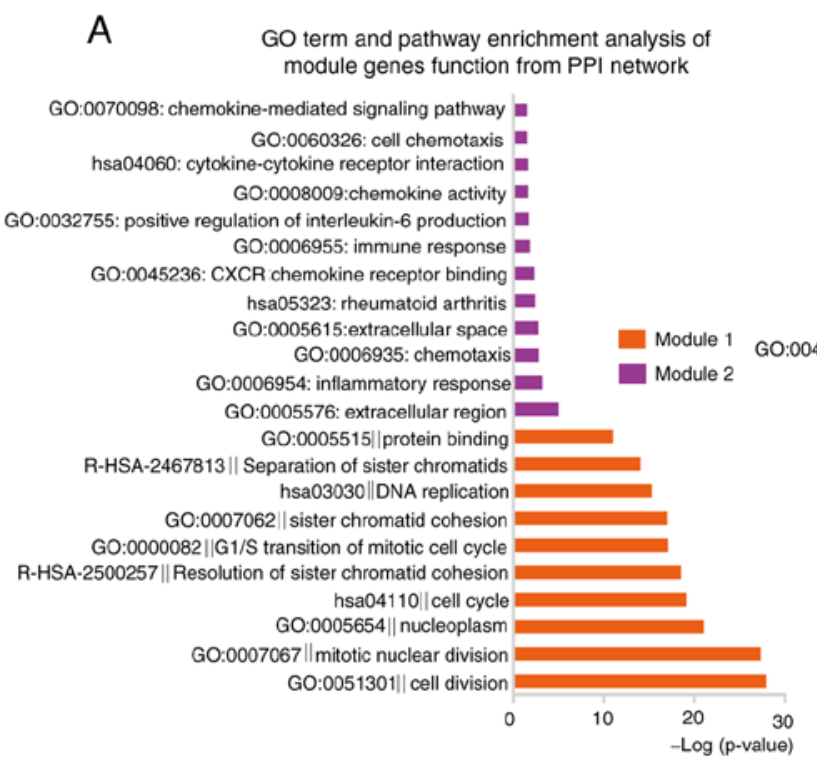

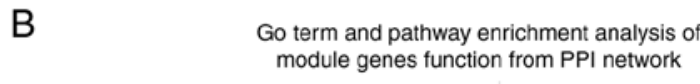

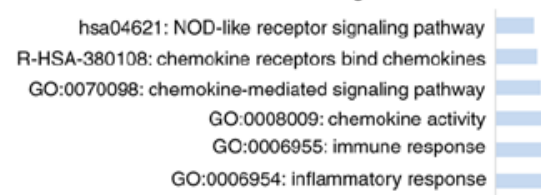

GO:0071356: cellular response to tumor necrosis factor

GO:0007155: cell adhesion
344: cellular response to fibroblast growth factor stimulus

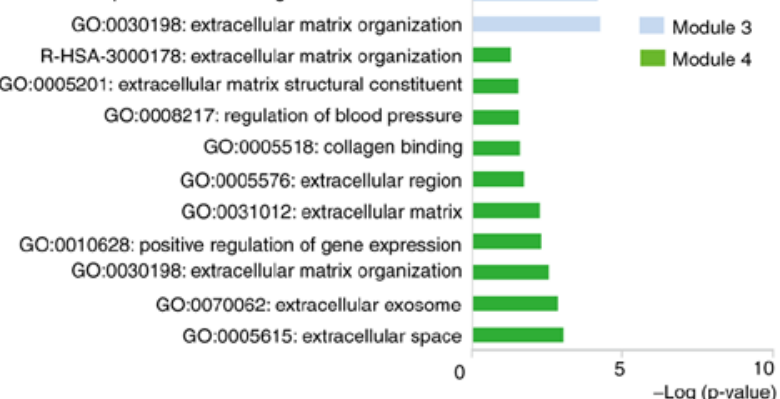

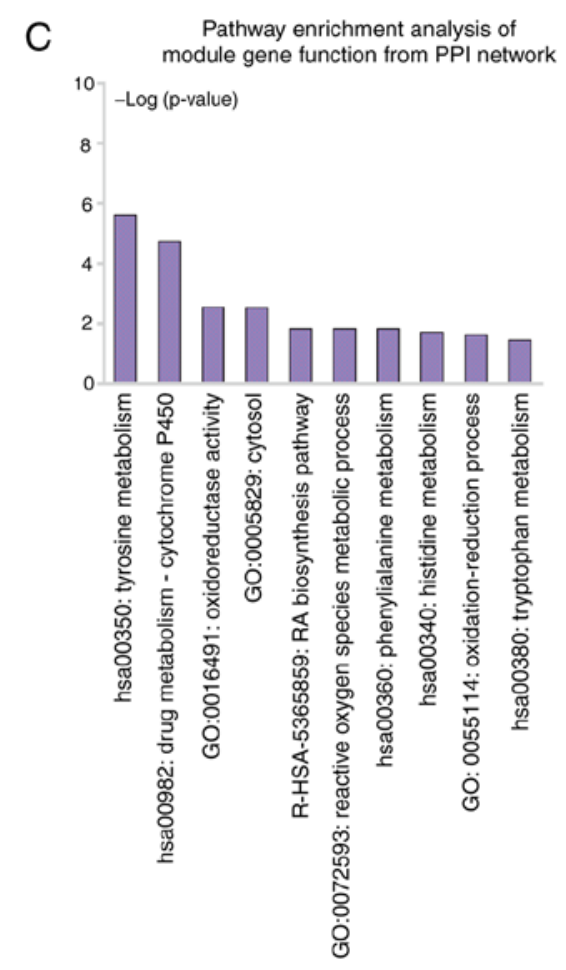

Figure 7. GO terms and pathways enrichment analysis of module genes from the PPI network. (A and B) GO terms and pathways enriched in the module genes in CVSMCs. (C) GO terms and pathways enriched in module genes between CVSMCs and calcifying osteoblasts. CVSMCs, calcifying vascular smooth muscle cells; PPI, protein-protein interaction; GO, Gene Ontology; Hsa, Homo sapiens.

transcription factor subunit, SNAI2, zinc finger and BTB domain containing 16, high mobility group AT-hook 1,CCAAT enhancer binding protein beta (CEBPB), E2F transcription factor 7 (E2F7), IL1B, forkhead box (FOX)M1, endothelial PAS domain protein 1 and forkhead box O1 (FOXO1) were differentially expressed and were used to construct a DETFmiRNA-target network, including 29 DETF-miRNAs and 27 miRNAs-target DEG pairs, such as FOXO1-hsa-miR-145, SNAI2-hsa-miR-221/222, SNAI2-hsa-miR-200a and SNAI2-hsa-mir-145 (Fig. 9B and Table SXIII). The hub genes in the PPI network that were potential targets of the predicted miRNAs, such as MAD2L1, KIF11, NCAPG, PBK, KIF23 and MELK, were incorporated into the TF-miRNA-hub gene network. The latter included $135 \mathrm{TF}-\mathrm{miRNA}$ and 13 miRNA-hub gene pairs, such as CEBPB-hsa-miR-20a-PBK, E2F7-hsa-miR-15a-KIF23 and RUNX family transcription factor 1 (RUNX1)-hsa-mir-181d-MELK (Fig. 9C and Table SXIV). Since CEBPB and E2F7 were identified as DETFs, the regulatory lncRNAs were next predicted. The putative CEBPB-targeting lncRNAs were metastasis associated lung adenocarcinoma transcript 1 , chondrogenesis-associated transcript, RAD51 antisense RNA 1 and negative regulator of antiviral response, whereas FOXF1, tensin 1, non-coding RNA activated by DNA damage and CDK6 antisense RNA 1 were predicted to regulate E2F7. In addition, lncRNAs regulating FOXO1 and SNAI2 were 


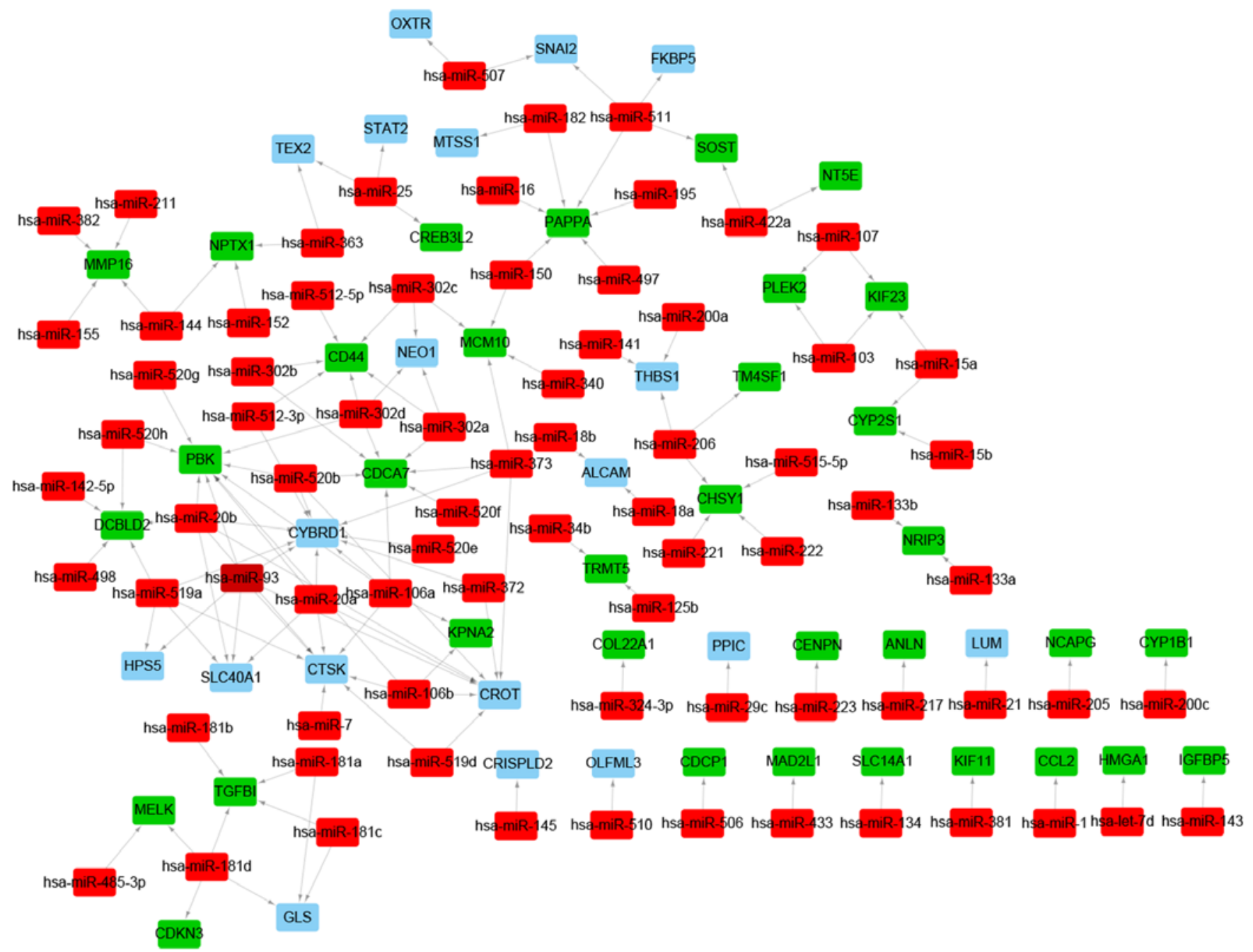

Figure 8. miRNA-target gene regulatory network. Red nodes represent miRNAs. Blue and green nodes represent the up- and downregulated target genes, respectively. Arrows indicate miRNA-targeting relationships. miRNA, microRNA; Hsa, Homo sapiens.

predicted. The lncRNA-DETF-miRNA-target gene regulatory network is presented in Fig. 9D.

Expression levels of certain potential crucial genes in VSMCs. To verify the in-silico results, a VSMC calcification model was established in vitro and the expression levels of selected relevant genes were analyzed. As presented in Fig. 10, differentiation antagonizing non-protein coding RNA (DANCR), MELK and FOXM1 were downregulated in the calcified VSMCs, while H19 imprinted maternally expressed transcript (H19), miR-485-3p, miR-181d, FOXO1 and SNAI2 were upregulated.

\section{Discussion}

In the present study, several putative genes, pathways and non-coding RNAs associated with VSMC calcification were identified, several of which were common to osteoblast mineralization. The DEGs in CVSMCs were mainly enriched in the cell cycle, ECM, inflammation and chemotaxis-mediated signaling pathways, whereas pathways related to the ECM, cytoplasm and metabolism were enriched among the DEGs shared between the CVSMCs and COs. The hub genes in the PPI network were MELK, PBK and KIF23, as well as DETFs including SNAI2, FOXM1 and FOXO1, and they are likely to regulate VSMC calcification.

In agreement with the results of the present bioinformatics analysis, previous studies using DNA microarray analysis of calcified aortic valve and proteomic analysis of calcified abdominal and thoracic aorta indicated that the DEGs were enriched in inflammation, chemokine and immune response signaling pathways $(29,30)$. In addition, chemokine expression was upregulated in the sclerotic aortic valves in an apolipoprotein E-deficient mouse model (31). The present results indicated that the ECM is similarly affected during VSMC and osteoblast mineralization, which is consistent with the increased mineral deposition observed in the ECM during VC. In addition, studies have revealed significant changes in the ECM proteins during VSMC calcification. For instance, collagen I and II content is markedly increased in calcified VSMCs and arteries (32-34). Collagen I may induce VSMC transdifferentiation into osteoblast-like cells and promote calcium crystallization by interacting with matrix vesicles $(32,35,36)$. Furthermore, degradation of ECM elastin also increases VSMC calcification and differentiation into osteoblast-like cells (37-39). By contrast, collagen IV expression decreased by $70 \%$ in calcified VSMCs, and collagen IV, 
A
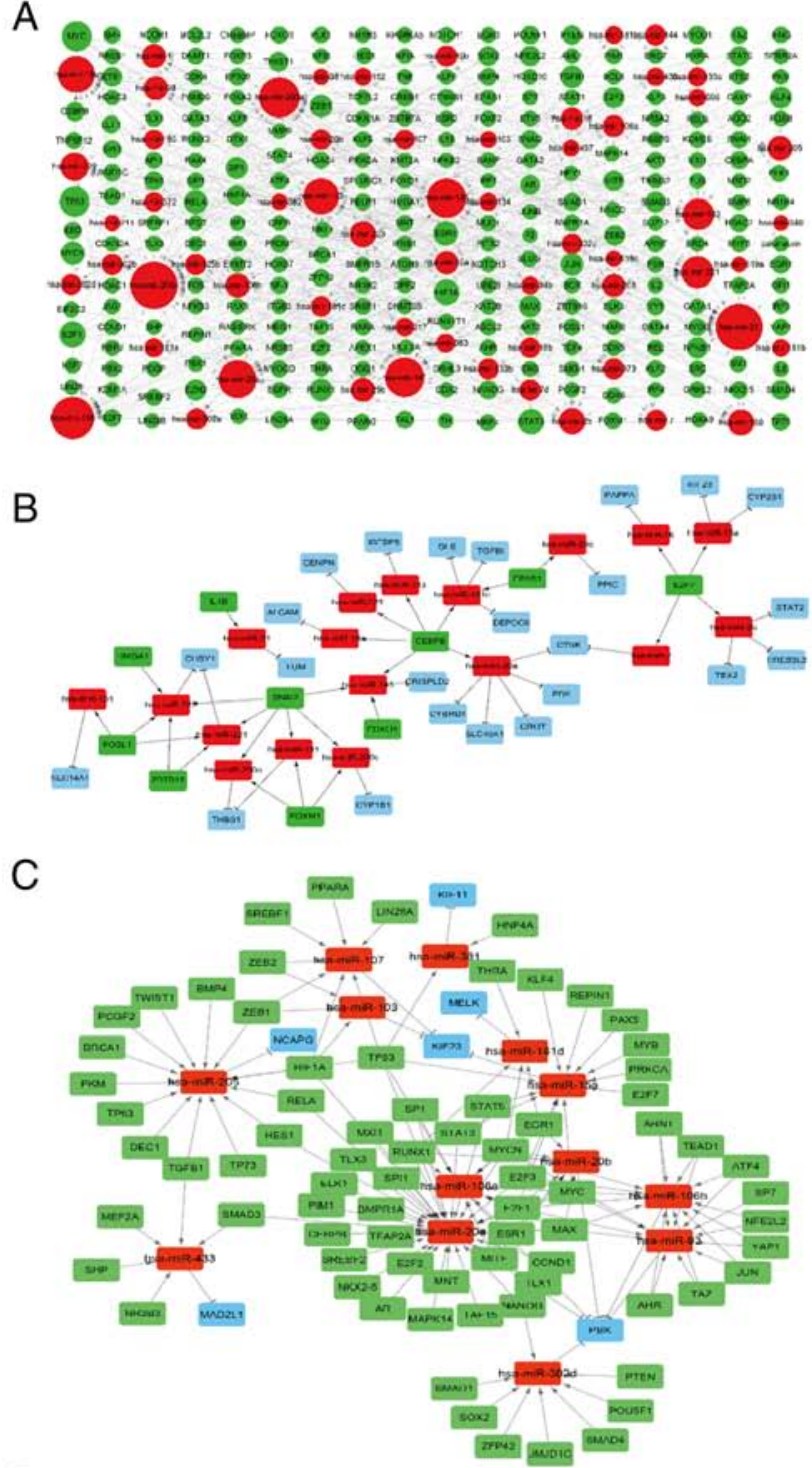

D

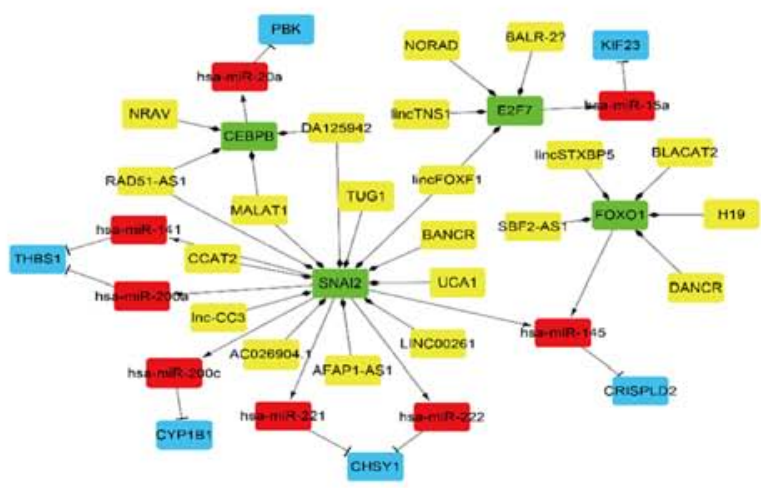

Figure 9. LncRNA-TF-miRNA-target gene regulatory network. (A) TF-miRNA regulatory network. Red and green nodes represent the miRNAs and TFs, respectively. The volume of gene nodes is proportional to the degree of connectivity of the gene nodes. (B) DETF-miRNA-target gene regulatory network. Red, green and blue nodes represent miRNAs, DETFs and DEGs, respectively. (C) TF-target miRNA-target hub gene regulatory network. Red, green and blue nodes represent miRNAs, TFs and target hub genes from the PPI network, respectively. (D) LncRNA-DETF-miRNA-target gene regulatory network. Yellow, green, red and blue nodes represen LncRNAs, DETFs, miRNAs and DEGs, respectively. Arrows, diamonds and ' $\mathrm{T}$ ' respectively indicate the TF-miRNA, lncRNA-target DETF and miRNA-target gene relationships. PPI, protein-protein interaction; TF, transcription factor; DETF, differentially expressed TF; lncRNA, long non-coding RNA; miRNA, microRNA; DEG, differentially expressed gene. collagen XIV and cartilage oligomeric matrix protein were all able to inhibit VSMC calcification and osteogenic differentiation through different signaling pathways $(35,36,40,41)$.

Several hub genes were identified in the PPI networks, several of which may regulate VSMC calcification. For instance, inhibition of MELK and its downstream target genes with the specific inhibitor OTSSP167 was reported to enhance osteoblast formation and matrix mineralization (42). In line with this, in the present study, MELK was downregulated in the CVSMCs, indicating that it is likely inhibited during VSMC calcification. The TFs FOXM1 and EZH2 are the downstream targets of MELK (43), of which FOXM1 was significantly downregulated in the present study. Dioscin-mediated inhibition of FOXM1 reduced VSMC proliferation and migration in vitro, as well as intimal thickening in a rat model with carotid artery balloon injury (44). EZH2 is also known to suppress osteogenic differentiation of mesenchymal cells and its inhibition promoted osteoblast differentiation $(45,46)$. Therefore, the MELK-FOXM1/EZH2 axis negatively regulates osteogenic differentiation and mineralization. MELK was also predicted as a target gene of hsa-miR-485-3p and hsa-miR-181d miRWalk (http://zmf.umm. uni-heidelberg.de; accessed April, 2019), indicating a novel has-485-3p/miR-181d-MELK-FOXM1/EZH2 axis (Fig. 11) in VSMC calcification that is worth exploring.

Other DETFs identified in the present study were FOXO1 and SNAI2. FOXO1 was previously reported to inhibit the osteogenic TF RUNX2 in prostate cancer cells $(47,48)$ and its inhibition in VSMCs prevented RUNX2 ubiquitination, which increased RNX2 levels and calcification (49). However, FOXO1 was determined to be upregulated in the present study, suggesting that it may also facilitate VSMC calcification. Consistent with this finding, FOXO1 levels were previously indicated to be increased in calcified femoropopliteal arteries from human subjects. Furthermore, five IncRNAs, including H19, DANCR, SBF2 antisense RNA 1, long intergenic non-protein coding syntaxin binding protein 5 and long intergenic non-protein coding RNA 958, were predicted as regulators of FOXO1 in this study. H19 downregulated FOXO1 in bovine skeletal muscle satellite cells, induced the osteogenic phenotype in valve interstitial cells and promoted aortic valve calcification by upregulating RUNX2 and bone morphogenetic protein (BMP)2, and inhibiting notch receptor $1(50,51)$. It is also upregulated during osteogenesis of human mesenchymal stem cells and promotes osteoblast differentiation via the Wnt/ $\beta$-catenin pathway (52). However, the role of the H19-FOXO1 axis in VSMC calcification and differentiation has remained elusive. DANCR regulates FOXO1 expression by affecting its ubiquitination (53). Zhu and $\mathrm{Xu}$ (54) and Jia et al (55) indicated that downregulation of DANC promoted osteogenic differentiation of human fetal osteoblastic cells and periodontal ligament stem cells, respectively. Therefore, it may be hypothesized that the DANCR-FOXO1 axis potentially regulates osteogenic differentiation of VSMCs. In addition, hsa-miR-145 is a potential biomarker of VC in chronic kidney disease (56) and was predicted as a target miRNA of FOXO1 in the DETF-miRNA-target regulatory network. Taken together, the H19/DANCR-FOXO1-miRNA-145-target gene axis has a crucial role during VSMC calcification and should be experimentally validated (Fig. 11). SNAI2 was predicted as 
A

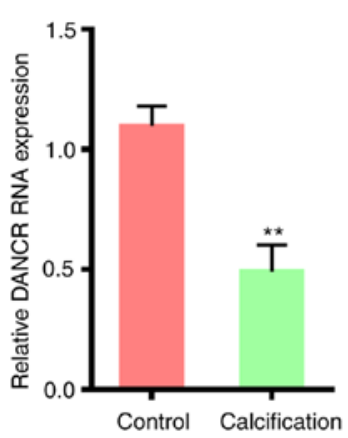

D

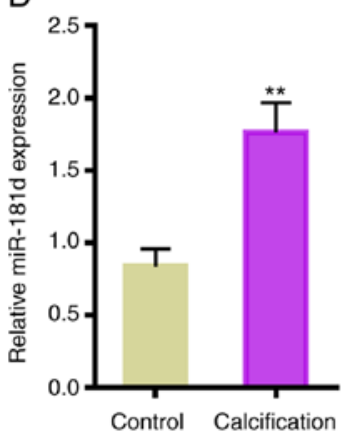

B

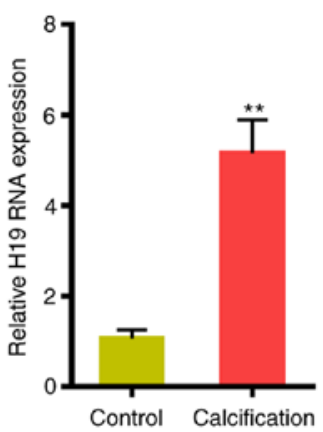

E

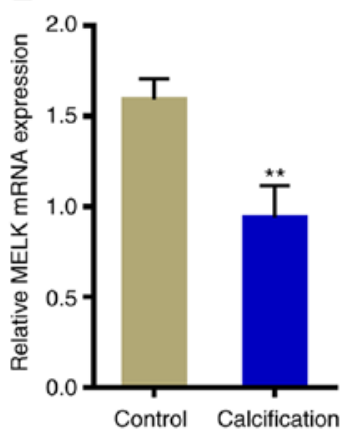

C

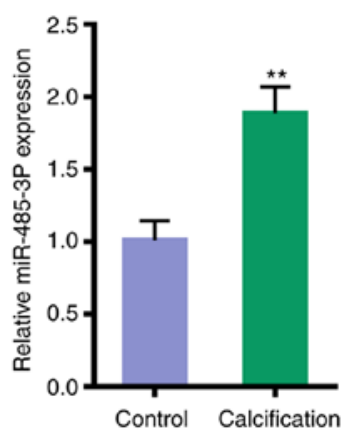

$\mathrm{F}$

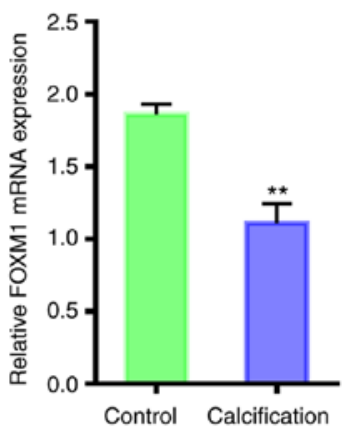

G

$\mathrm{H}$
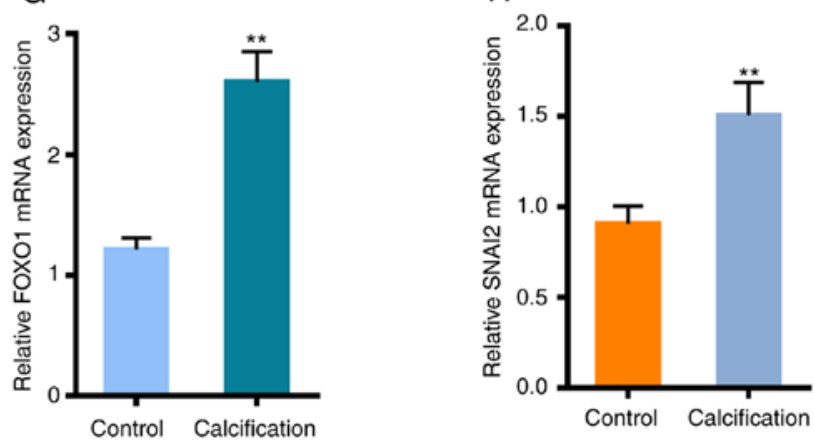

Figure 10. Validation of crucial genes in CVSMCs. Expression levels of (A) DANCR, (B) H19, (C) miR-485-3p, (D) miR-181d, (E) MELK, (F) FOXM1, (G) FOXO1 and (H) SNAI2 in the normal VSMCs and CVSMCs. ${ }^{* *} \mathrm{P}<0.05$ vs. control. CVSMCs, calcifying vascular smooth muscle cells; miR, microRNA; FOX, forkhead box; DANCR, differentiation antagonizing non-protein coding RNA; H19, H19 imprinted maternally expressed transcript; MELK, maternal embryonic leucine zipper kinase; SNAI2, snail family transcriptional repressor 2.

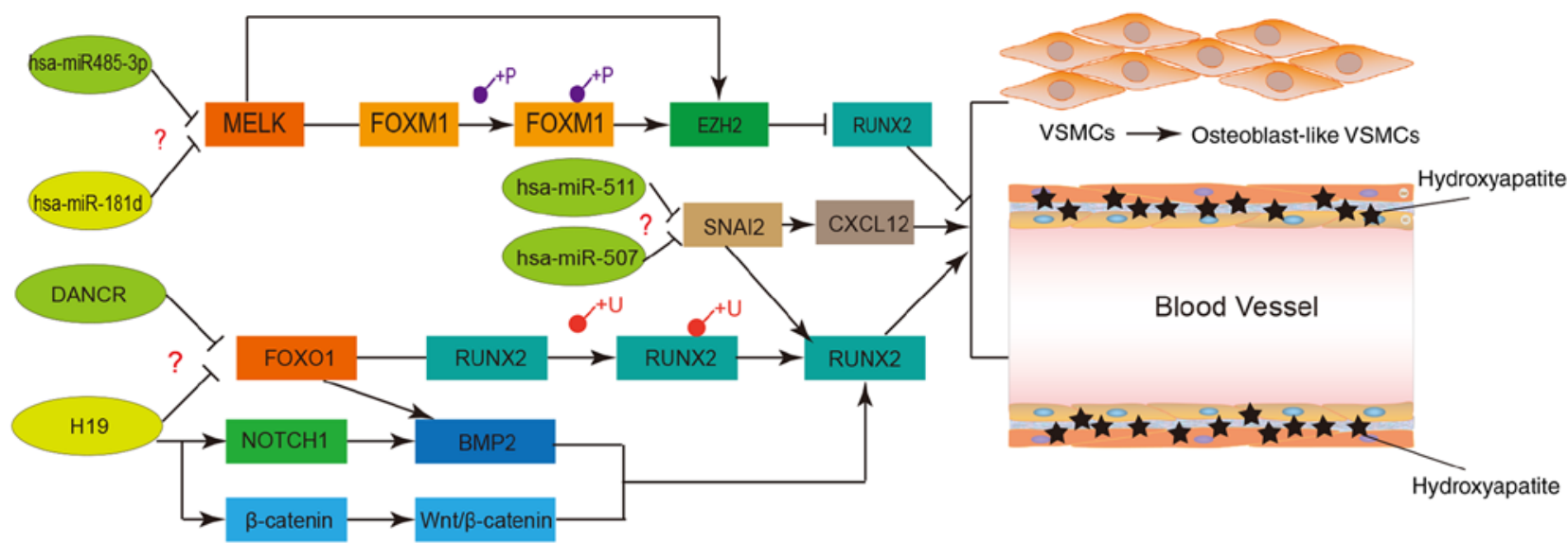

Figure 11. Potential regulatory networks and pathways of certain crucial long non-coding RNAs, miRNAs and proteins involved in VSMC calcification VSMCs, vascular smooth muscle cells; miRNA/miR, microRNA; FOX, forkhead box; DANCR, differentiation antagonizing non-protein coding RNA; H19, H19 imprinted maternally expressed transcript; MELK, maternal embryonic leucine zipper kinase; SNAI2, snail family transcriptional repressor 2.RUNX2, RUNX family transcription factor 2; CXCL12, C-X-C motif chemokine ligand 12; NOTCH1, notch receptor 1; EZH2, enhancer of zeste 2 polycomb repressive complex 2 subunit; BMP2, bone morphogenetic protein $2 ; \beta$-catenin, catenin beta interacting protein 1. 
a target of six miRNAs. Previous studies indicated that SNAI2 promotes osteoblast maturation by upregulating RUNX2, as well as osteoblast mineralization through $\mathrm{C}-\mathrm{X}-\mathrm{C}$ motif chemokine ligand 12 signaling $(57,58)$. In addition, SNAI2 also mediated BMP-dependent transdifferentiation of mouse non-ciliated aortic endothelial cells into mineralizing osteogenic cells and promoted atherosclerosis and $\mathrm{VC}$ in vivo (59). In line with this, in the present study, the upregulation of SNAI2 suggested its stimulatory effect on VSMC calcification. Furthermore, hsa-miR-511 and hsa-miR-507, as well as 12 lncRNAs, were predicted to regulate SNAI2. Most of these lncRNAs were identified in tumors. Therefore, it was hypothesized that the hsa-miR-511/hsa-miR-507-SNAI2 axis and the lncRNAs-SNAI2 axis potentially regulate VSMC calcification and should be explored further (Fig. 11).

In conclusion, the present study identified several potential regulatory mechanisms of VC and the DEGs and signaling pathways associated with the calcification of VSCMs. They involve changes in the inflammatory response, chemotaxis and ECM, and the latter is characteristic of osteoblast mineralization as well. Mechanistically, the hsa-485-3p/miR-181d-MELKFOXM1/EZH2, H19/DANCR-FOXO1 and SNAI2-mediated pathways possibly regulate $\mathrm{VC}$. These mechanisms require to be experimentally validated in future studies.

\section{Acknowledgements}

Not applicable.

\section{Funding}

This work was supported in part by the self-financing research project of the Health and Family Planning Commission of Guangxi Zhuang Autonomous Region (grant no. Z20180518).

\section{Availability of data and materials}

The data sets used and/or analyzed during the current study are available from the corresponding author on reasonable request. In addition, the dataset GSE37558 may be obtained from the GEO database (https://www.ncbi.nlm.nih.gov/geo/query/acc. cgi?acc=GSE37558).

\section{Authors' contributions}

$\mathrm{XW}, \mathrm{YS}, \mathrm{QL}, \mathrm{ZZ}$ and $\mathrm{PH}$ contributed to the study conception and design. PH, XW and YS checked the associated databases and analyzed raw data for bioinformatics analysis, cell culture, PCR. XW, QL and ZZ wrote and revised the manuscript. All of the authors read and approved the final manuscript. $\mathrm{PH}$ and XW checked and approved the authenticity of the raw data. All authors read and approved the final manuscript.

\section{Ethics approval and consent to participate}

Not applicable.

\section{Patient consent for publication}

Not applicable.

\section{Competing interests}

The authors declare that they have no competing interests.

\section{References}

1. Lanzer P, Boehm M, Sorribas V, Thiriet M, Janzen J, Zeller T, St Hilaire C and Shanahan C: Medial vascular calcification revisited: Review and perspectives. Eur Heart J 35: 1515-1525, 2014.

2. Kovacic JC and Fuster V: Vascular calcification, diabetes, and cardiovascular disease: Connecting the dots. JACC Cardiovasc Imaging 5: 367-369, 2012.

3. Chen NX and Moe SM: Pathophysiology of vascular calcification. Curr Osteoporos Rep 13: 372-380, 2015.

4. Leopold JA: Vascular calcification: Mechanisms of vascular smooth muscle cell calcification. Trends Cardiovasc Med 25: 267-274, 2015.

5. Block GA, Spiegel DM, Ehrlich J, Mehta R, Lindbergh J, Dreisbach A and Raggi P: Effects of sevelamer and calcium on coronary artery calcification in patients new to hemodialysis. Kidney Int 68: 1815-1824, 2005.

6. Blomberg BA, de Jong PA, Thomassen A, Lam MGE, Vach W, Olsen MH, Mali WPTM, Narula J, Alavi A and Høilund-Carlsen PF: Thoracic aorta calcification but not inflammation is associated with increased cardiovascular disease risk: Results of the CAMONA study. Eur J Nucl Med Mol Imaging 44: 249-258, 2017.

7. Bastos Gonçalves F, Voûte MT, Hoeks SE, Chonchol MB Boersma EE, Stolker RJ and Verhagen HJ: Calcification of the abdominal aorta as an independent predictor of cardiovascular events: A meta-analysis. Heart 98: 988-994, 2012.

8. Bild DE, Detrano R, Peterson D, Guerci A, Liu K, Shahar E, Ouyang P, Jackson S and Saad MF: Ethnic differences in coronary calcification: The Multi-Ethnic Study of Atherosclerosis (MESA). Circulation 111: 1313-1320, 2005.

9. Zhang MJ, Zhou Y, Chen L, Wang YQ, Wang X, Pi Y, Gao CY, Li JC and Zhang LL: An overview of potential molecular mechanisms involved in VSMC phenotypic modulation. Histochem Cell Biol 145: 119-130, 2016.

10. Gomez D and Owens GK: Smooth muscle cell phenotypic switching in atherosclerosis. Cardiovasc Res 95: 156-164, 2012.

11. Kapustin AN and Shanahan CM: Calcium regulation of vascular smooth muscle cell-derived matrix vesicles. Trends Cardiovasc Med 22: 133-137, 2012.

12. Yao RW, Wang Y and Chen LL: Cellular functions of long noncoding RNÂs. Nat Cell Biol 21: 542-551, 2019.

13. Bar C, Chatterjee $\mathrm{S}$ and Thum T: Long noncoding RNAs in cardiovascular pathology, diagnosis, and therapy. Circulation 134: 1484-1499, 2016

14. Barwari T, Joshi A and Mayr M: MicroRNAs in cardiovascular disease. J Am Coll Cardiol 68: 2577-2584, 2016.

15. Haemmig S, Simion V, Yang D, Deng Y and Feinberg MW: Long noncoding RNAs in cardiovascular disease, diagnosis, and therapy. Curr Opin Cardiol 32: 776-783, 2017.

16. Jiang W, Zhang Z, Yang H, Lin Q, Han C and Qin X: The involvement of miR-29b-3p in arterial calcification by targeting matrix metalloproteinase-2. Biomed Res Int 2017: 6713606, 2017.

17. Cui RR, Li SJ, Liu LJ, Yi L, Liang QH, Zhu X, Liu GY, Liu Y, Wu SS, Liao XB, et al: MicroRNA-204 regulates vascular smooth muscle cell calcification in vitro and in vivo. Cardiovasc Res 96: 320-329, 2012.

18. Ding W, Li J, Singh J, Alif R, Vazquez-Padron RI, Gomes SA, Hare JM and Shehadeh LA: miR-30e targets IGF2-regulated osteogenesis in bone marrow-derived mesenchymal stem cells, aortic smooth muscle cells, and ApoE 2/2 mice. Cardiovasc Res 106: 131-142, 2015.

19. Du Y, Gao C, Liu Z, Wang L, Liu B, He F, Zhang T, Wang Y, Wang X, Xu M, et al: Upregulation of a disintegrin and metalloproteinase with thrombospondin motifs- 7 by miR-29 repression mediates vascular smooth muscle calcification. Arterioscler Thromb Vasc Biol 32: 2580-2588, 2012.

20. Wang XY, Zhang XZ, Li F and Ji QR: MiR-128-3p accelerates cardiovascular calcification and insulin resistance through ISL1-dependent Wnt pathway in type 2 diabetes mellitus rats. J Cell Physiol 234: 4997-5010, 2019. 
21. Lin X, Zhan JK, Zhong JY, Wang YJ, Wang Y, Li S, He JY, Tan P, Chen YY, Liu XB, et al: lncRNA-ES3/miR-34c-5p/BMF axis is involved in regulating high-glucose-induced calcification/senescence of VSMCs. Aging (Albany NY) 11: 523-535, 2019.

22. Jeong G, Kwon DH, Shin S, Choe N, Ryu J, Lim YH, Kim J, Park WJ, Kook H and Kim YK: Long noncoding RNAs in vascular smooth muscle cells regulate vascular calcification. Sci Rep 9: 5848, 2019.

23. Alves RD, Eijken M, van de Peppel J and van Leeuwen JP: Calcifying vascular smooth muscle cells and osteoblasts: Independent cell types exhibiting extracellular matrix and biomineralization-related mimicries. BMC Genomics 15: 965, 2014.

24. Karagoz K, Sevimoglu T and Arga KY: Integration of multiple biological features yields high confidence human protein interactome. J Theor Biol 403: 85-96, 2016.

25. Shannon P, Markiel A, Ozier O, Baliga NS, Wang JT, Ramage D, Amin N, Schwikowski B and Ideker T: Cytoscape: A software environment for integrated models of biomolecular interaction networks. Genome Res 13: 2498-2504, 2003.

26. Tong Z, Cui Q, Wang J and Zhou Y: TransmiR v2.0: An updated transcription factor-microRNA regulation database. Nucleic Acids Res 47: D253-D258, 2019.

27. Cheng L, Wang P, Tian R, Wang S, Guo Q, Luo M, Zhou W, Liu G, Jiang H and Jiang Q: LncRNA2Target v2 .0: A comprehensive database for target genes of lncRNAs in human and mouse. Nucleic Acids Res 47: D140-D144, 2019.

28. Livak KJ and Schmittgen TD: Analysis of relative gene expression data using real-time quantitative PCR and the 2(-Delta Delta C(T)) method. Method 25: 402-408, 2001.

29. Ohukainen P, Syväranta S, Näpänkangas J, Rajamäki K, Taskinen P, Peltonen T, Helske-Suihko S, Kovanen PT, Ruskoaho H and Rysä J: MicroRNA-125b and chemokine CCL4 expression are associated with calcific aortic valve disease. Ann Med 47: 423-429, 2015.

30. Matsumoto K, Maniwa T, Tanaka T, Satoh K, Okunishi H and Oda T: Proteomic analysis of calcified abdominal and thoracic aortic aneurysms. Int J Mol Med 30: 417-429, 2012.

31. Tanaka K, Sata M, Fukuda D, Suematsu Y, Motomura N, Takamoto S, Hirata Y and Nagai R: Age-associated aortic stenosis in apolipoprotein E-deficient mice. J Am Coll Cardiol 46: 134-141, 2005.

32. Hodroge A, Trécherel E, Cornu M, Darwiche W, Mansour A, Ait-Mohand K, Verissimo T, Gomila C, Schembri C, Da Nascimento $S$, et al: Oligogalacturonic acid inhibits vascular calcification by two mechanisms: inhibition of vascular smooth muscle cell osteogenic conversion and interaction with collagen. Arterioscler Thromb Vasc Biol 37: 1391-1401, 2017.

33. Shanahan CM, Cary NR, Salisbury JR, Proudfoot D, Weissberg PL and Edmonds ME: Medial localization of mineralization-regulating proteins in association with Mönckeberg's sclerosis. Circulation 100: 2168-2176, 1999.

34. Tyson KL, Reynolds JL, McNair R, Zhang Q, Weissberg PL and Shanahan CM: Osteo/chondrocytic transcription factors and their target genes exhibit distinct patterns of expression in human arterial calcification. Arterioscler Thromb Vasc Biol 23 489-494, 2003.

35. Watson KE, Parhami F, Shin V and Demer LL: Fibronectin and collagen I matrixes promote calcification of vascular cells in vitro, whereas collagen IV matrix is inhibitory. Arterioscler Thromb Vasc Biol 18: 1964-1971, 1998.

36. Freise C, Bobb V and Querfeld U: Collagen XIV and a related recombinant fragment protect human vascular smooth muscle cells from calcium-/phosphate-induced osteochondrocytic transdifferentiation. Exp Cell Res 358: 242-252, 2017

37. Lei Y, Sinha A, Nosoudi N, Grover A and Vyavahare N: Hydroxyapatite and calcified elastin induce osteoblast-like differentiation in rat aortic smooth muscle cells. Exp Cell Res 323: 198-208, 2014.

38. Sinha A and Vyavahare NR: High-glucose levels and elastin degradation products accelerate osteogenesis in vascular smooth muscle cells. Diabet Vasc Dis Res 10: 410-419, 2013.

39. Urry DW: Neutral sites for calcium ion binding to elastin and collagen: A charge neutralization theory for calcification and its relationship to atherosclerosis. Proc Natl Acad Sci USA 68: 810-814, 1971.

40. Wang L, Zheng J, Du Y, Huang Y, Li J, Liu B, Liu CJ, Zhu Y, Gao Y, Xu Q, et al: Cartilage oligomeric matrix protein maintains the contractile phenotype of vascular smooth muscle cells by interacting with alpha(7)beta(1) integrin. Circ Res 106: 514-525, 2010.
41. Du Y, Wang Y, Wang L, Liu B, Tian Q, Liu CJ, Zhang T, Xu Q, Zhu Y, Ake O, et al: Cartilage oligomeric matrix protein inhibits vascular smooth muscle calcification by interacting with bone morphogenetic protein-2. Circ Res 108: 917-928, 2011.

42. Muller J, Bolomsky A, Dubois S, Duray E, Stangelberger K, Plougonven E, Lejeune M, Léonard A, Marty C, Hempel U, et al: Maternal embryonic leucine zipper kinase inhibitor OTSSP167 has preclinical activity in multiple myeloma bone disease. Haematologica 103: 1359-1368, 2018.

43. Kim SH, Joshi K, Ezhilarasan R, Myers TR, Siu J, Gu C, Nakano-Okuno M, Taylor D, Minata M, Sulman EP, et al: EZH2 protects glioma stem cells from radiation-induced cell death in a MELK/FOXM1-dependent manner. Stem Cell Reports 4: 226-238, 2015.

44. Fan T, He J, Yin Y, Wen K, Kang Y, Zhao H, Chen S and Li X: Dioscin inhibits intimal hyperplasia in rat carotid artery balloon injury model through inhibition of the MAPK-FoxM1 pathway. Eur J Pharmacol 854: 213-223, 2019.

45. Dudakovic A, Camilleri ET, Xu F, Riester SM, McGeeLawrence ME, Bradley EW, Paradise CR, Lewallen EA, Thaler R, Deyle DR, et al: Epigenetic control of skeletal development by the histone methyltransferase Ezh2. J Biol Chem 290: 27604-27617, 2015.

46. Adamik J, Jin S, Sun Q, Zhang P, Weiss KR, Anderson JL, Silbermann R, Roodman GD and Galson DL: EZH2 or HDAC1 inhibition reverses multiple myeloma-induced epigenetic suppression of osteoblast differentiation. Mol Cancer Res 15: 405-417, 2017.

47. Zhang H, Pan Y, Zheng L, Choe C, Lindgren B, Jensen ED, Westendorf JJ, Cheng L and Huang H: FOXO1 inhibits Runx2 transcriptional activity and prostate cancer cell migration and invasion. Cancer Res 71: 3257-3267, 2011

48. Sun Y, Byon CH, Yuan K, Chen J, Mao X, Heath JM, Javed A, Zhang K, Anderson PG and Chen Y: Smooth muscle cell-specific Runx2 deficiency inhibits vascular calcification. Circ Res 111: 543-552, 2012.

49. Deng L, Huang L, Sun Y, Heath JM, Wu H and Chen Y: Inhibition of FOXO1/3 promotes vascular calcification. Arterioscler Thromb Vasc Biol 35: 175-183, 2015

50. Xu X, Ji S, Li W, Yi B, Li H, Zhang H and Ma W: LncRNA H19 promotes the differentiation of bovine skeletal muscle satellite cells by suppressing Sirt1/FoxO1. Cell Mol Biol Lett 22: 10, 2017.

51. Hadji F, Boulanger MC, Guay SP, Gaudreault N, Amellah S, Mkannez G, Bouchareb R, Marchand JT, Nsaibia MJ, Guauque-Olarte $S$, et al: Altered DNA methylation of long noncoding RNA H19 in calcific aortic valve disease promotes mineralization by silencing NOTCH1. Circulation 134: 1848-1862, 2016.

52. Liang WC, Fu WM, Wang YB, Sun YX, Xu LL, Wong CW, Chan KM, Li G, Waye MM and Zhang JF: H19 activates Wnt signaling and promotes osteoblast differentiation by functioning as a competing endogenous RNA. Sci Rep 6: 20121, 2016.

53. Tang Z, Gong Z and Sun X: LncRNA DANCR involved osteolysis after total hip arthroplasty by regulating FOXO1 expression to inhibit osteoblast differentiation. J Biomed Sci 25: 4, 2018.

54. Zhu L and Xu PC: Downregulated LncRNA-ANCR promotes osteoblast differentiation by targeting EZH2 and regulating Runx2 expression. Biochem Biophys Res Commun 432: 612-617, 2013.

55. Jia Q, Jiang W and Ni L: Down-regulated non-coding RNA (lncRNA-ANCR) promotes osteogenic differentiation of periodontal ligament stem cells. Arch Oral Biol 60: 234-241, 2015.

56. Massy ZA, Metzinger-Le Meuth V and Metzinger L: MicroRNAs are associated with uremic toxicity, cardiovascular calcification, and disease. Contrib Nephrol 189: 160-168, 2017.

57. Lambertini E, Lisignoli G, Torreggiani E, Manferdini C, Gabusi E, Franceschetti T, Penolazzi L, Gambari R, Facchini A and Piva R: Slug gene expression supports human osteoblast maturation. Cell Mol Life Sci 66: 3641-3653, 2009.

58. Piva R, Manferdini C, Lambertini E, Torreggiani E, Penolazzi L, Gambari R, Pastore A, Pelucchi S, Gabusi E, Piacentini A, et al: Slug contributes to the regulation of CXCL12 expression in human osteoblasts. Exp Cell Res 317: 1159-1168, 2011.

59. Sanchez-Duffhues G, de Vinuesa AG, Lindeman JH, Mulder-Stapel A, DeRuiter MC, Van Munsteren C, Goumans MJ, Hierck BP and Ten Dijke P: SLUG is expressed in endothelial cells lacking primary cilia to promote cellular calcification. Arterioscler Thromb Vasc Biol 35: 616-627, 2015.

This work is licensed under a Creative Commons Attribution-NonCommercial-NoDerivatives 4.0 International (CC BY-NC-ND 4.0) License. 